\title{
Guest Editorial: Special Issue of ESREL2020 PSAM15
}

\author{
Francesco Di Maio ${ }^{1}$ Enrico Zioo,
}

Published online: 12 February 2022

(o) The Author(s), under exclusive licence to Springer Nature Switzerland AG 2022

The Conference, originally planned to be held in Venice, Italy, has eventually been held remotely on November 1-5, 2020. The event has combined the 30th European Safety and Reliability Conference (ESREL 2020) and the 15th Probabilistic Safety Assessment and Management Conference (PSAM 15). Such Conference series is the largest and most important for the scientific community of the areas of reliability, safety and security. Previous events have included Crete 1996, Berlin 2004, Helsinki 2012. The Conference provides a forum for discussion of advancements in the development of scientific methodologies and deployment of technical solutions for the reliable design and operation of components and systems, and for the prevention and management of risk in complex systems and critical infrastructures.

In this edition, the program of the Conference has consisted in 728 abstracts and papers selected through a peer-review process conducted by more than 130 Track Directors, who have organized the work of more than 800 reviewers. The program has been enriched by 10 plenary lectures offered by speakers of international excellence, 5 panels, 11 special sessions and 2 innovation challenges.

The Conference works have covered 55 topics and 31 application areas: this special issue presents selected works from the sessions on Cyber Security, External Hazards Risk Assessment, Security, Software Reliability and Safety and Web Security.

The unifying motivation behind the selection of these specific works is that of reporting on the international advancements in security-related multi-disciplinary research, highlighting their impact on diverse critical infrastructures such as those

The present special issue contains extended, representative works, selected from those presented at the ESREL 2020 PSAM 15 Conference.

Francesco Di Maio

francesco.dimaio@polimi.it

Enrico Zio

enrico.zio@polimi.it

1 Energy Department, Politecnico Di Milano, Via La Masa 34, 20126 Milan, Italy

2 Centre de Recherche Sur Les Risques Et Les Crises (CRC), MINES ParisTech / PSL Université Paris, Sophia Antipolis, France 
devoted to energy production/transmission and distribution, transportation and information and communication.

The first paper by Pengyu Zhu and Jayantha P. Liyanage is a review paper on opportunities and challenges brought by the digitalization of Safety Instrumented Systems (SIS) in offshore Oil\&Gas (O\&G) production assets. A SIS plays a critical role to guarantee a specific Safety Integrity Level (SIL) of the asset, since it consists of specific instrumentation to detect, prevent, and mitigate hazardous situations for process safety. The concerns related to the effects of digital technologies-driven developments of SIS are discussed with respect to safety and security in a regulatory perspective. The rationale is that both SIS hardware and software upgrades to digital systems can expose an $\mathrm{O} \& \mathrm{G}$ production asset to new threats and risks: in particular, authorities, operators and service providers should be particularly sensitive and attentive to cyber-attacks. In the paper, requirements for standards and guidelines are drawn, pointing out a list of dangerous failure modes of SIS, that require due security-oriented attention in a continuous asset digitalization process. Finally, specific issues and challenges related to cybersecurity of SIS are discussed.

The second paper by Hossein Hassani, Roozbeh Razavi-Far, Mehrdad Saif and Enrico Zio presents an approach based on deep learning with long short-term memory networks for diagnosing faults in smart grids and avoid cascading failures that can lead to blackout. The proposed approach makes use of the voltage measurements collected from different spots in the system that are, then, fed into a signal processing module. Within the module, matching pursuit decomposition and variational mode decomposition are used to extract a set of discriminative features that are, then, fed to a classification module that employs long short-term memory networks for fault diagnosis. The applicability and effectiveness of the proposed diagnostic framework is shown with respect to various simulated scenarios that may occur in the IEEE 39-bus system.

The third paper by Jo Laufenberg, Thomas Kropf and Oliver Bringmann, proposes a method for the cyber-attack detection in the Controller Area Network (CAN) of modern cars. The need originates from the fact that CAN is still highly used despite no security features are devised, exposing the cars to remote attacks. Only an Intrusion Detection System (IDS) can secure the communication. In the paper, the proposed method for IDS monitors the CAN communications and uses static checks to differentiate between normal and attack traffic. Results show that the IDS achieves a zero false alarms rate for the investigated data sets.

The fourth paper by Daniel Lichte, Dustin Witte, Thomas Termin and KaiDietrich Wolf addresses the relevant aspects of uncertainty representation and quantification to support quantitative physical Security Risk Assessment (SRA) of critical infrastructures. Due to little evidence of actual attacks, the availability of vague data and the massive reliance to expert knowledge, quantitative risk assessment approaches suffer from the fundamental problem of uncertainty quantification regarding threats and capabilities of security measures. In the paper, the authors propose the concept of security margin that accounts for the uncertain knowledge of the input parameters in the design of a security system and minimizes the influence of the uncertainty on the actual system performance. On a case study that concerns an airport critical infrastructure, the authors show i) the effects of uncertainty on 
the results, by comparing a quantitative SRA that neglects uncertainty with a SRA whose inputs uncertainty is quantified and ii) that the security margin quantification can help reducing the system vulnerability.

The fifth paper by Fabrizio Baiardi and Federico Tonelli discusses the role of digital twins to minimize cyber risks of an information and communication infrastructure. Digital twins are virtual replicas of real physical devices: the authors propose to couple the digital twin of the infrastructure with an adversary threat emulator to define a remediation policy that selects and schedules patches for the vulnerabilities before the attackers can breach the information and telecommunication infrastructure. The Haruspex software platform builds the twins of the infrastructure and those of the attackers: the twin-based approach supports a continuous remediation process to handle changes in the infrastructure, new vulnerabilities and new threats, since the platform can update the twins and run adversary emulations. The effectiveness of the proposal is supported by the results on experimental data.

In closing this guest editorial, we would like to thank the authors for their outstanding contributions and the reviewers for their hard, timely and professional work. We also wish to acknowledge that this special issue would have not been possible without the kind support of Prof. Stefan Kaufmann, Editor-in-Chief of the journal, who has given us the opportunity and the assistance necessary to put together such a collection of interesting works. To all these people goes our sincere professional appreciation and personal gratitude.

Publisher's Note Springer Nature remains neutral with regard to jurisdictional claims in published maps and institutional affiliations. 